\title{
MOTIVATION FOR EDUCATION AND LEARNERS' SATISFACTION WITH THE CHOICE OF MILITARY SPECIALISATION OR CIVILIAN SPECIALTY
}

\author{
Elitsa PETROVA \\ "Vasil Levski" National Military University, Veliko Tarnovo, Bulgaria \\ elitsasd@abv.bg
}

\begin{abstract}
This article presents a research on the motivation for education and training activities and their relation with the satisfaction of choosing a military specialisation or a civilian specialty, based on the example of cadets and students from the Vasil Levski National Military University in Bulgaria. Motivation for education and training activities is the main research subject of the study which is conducted in a real educational environment. The object of the study is cadets in the last year of their education in the Military Science professional field, specialising in Organisation and Management of Military Units at a Tactical Level at the National Military University in Bulgaria. The study includes students and cadets in the first year of education as control groups for comparison. The study launched in 2012. It continues to the present. It is supported by military experts from over 11 foreign military educational institutions - universities and academies from all across Europe.
\end{abstract}

Keywords: motivation, education, military, civil

\section{Introduction}

Motivational factors are impacts that the management uses and applies to affect individual behaviour on the labour market and in daily routine. They are a complex combination of economic, organisational, managerial, social and psychological factors which affect job performance and the results of the staff's work activity. The skilful use of motivational tools makes modern organisations competitive.[4,5,6] The subject of this scientific study is the motivation for education and training, and the objects are cadets in several consecutive years in the Military Science professional field, specialising in Organisation and Management of Military Units at a Tactical Level at the National Military University in Bulgaria. Students and cadets in the first year of their training are included as control groups for comparison. The study on learners' motivation goes through several phases - diagnostic, basic and final. This paper presents parts of the research conducted in the basic stage.

\section{Scientific Methodology for Studying the Motivation for Education and Some Specific Military Activities in the Learning Process}

The basic part of the study of the motivation for education and some specific military activities in the learning process is conducted in the period 2012-2016 and continues to date. It aims to cover at least four consecutive years of cadets. [7,8,9] The general statistical collection of respondents includes about and over $85 \%$ of trainees in different academic years. The study uses a questionnaire of 24 questions. The answers can be given, depending on theasked questions, by one of the following fivepoint scale:

- To expresssatisfaction, dissatisfaction or indifference, the answers can be given in the following scale: to a very large extent; to a large extent; neither to a 
small, nor to a large extent; to a small extent and to a very small extent.

- To express consent, dissent or indifference, the answers can be given in the following scale: totally agree; agree; without relevance/doesn't matter; disagree and totally disagree.

The questionnaire should be filled in individually by each participant in the study. It was explained in advance that the questions were exploratory in nature and filling the questionnaire would not lead to sanctions and penalties to the participant, which promoted freedom in expressing their personal opinion.

The extent of satisfaction of the needs of the surveyed learners is determined in processing the responses. The answers give direct opportunity to establish satisfaction, indifference and dissatisfaction as well as consent, indifference and dissent on different motivational elements. All tested items are visualized by graphs, tables, charts and models of trends.

During the basic stage of the study the following were conducted:

- Research on motivation for training and military activities in the learning process and research on satisfaction using 24 questions for the main target group cadets who graduated in the academic years 2013/2014, 2014/2015 and 2015/2016 - 144 respondents.

- Research on motivation for training in the learning process and research on satisfaction using 15 questions for the control group for comparison which included first-year cadets who started their training in 2013/2014 - 77 respondents.

- Research on motivation for training in the learning process and research on satisfaction using 15 questions for the control group for comparison which included first-year civilian students who started their training in 2013/2014 - 88 respondents.
3. Motivation for education and its relationship with satisfaction with the choice of academic specialty

All individuals are motivated to work for an organisation where they are accepted, understood, and capable to establish themselves as individuals and professionals, and can influence other individuals and events.[1,2] The satisfaction from any human activity reflects the nature of feelings that people have, including the results of the activity. Satisfaction is the attitude which determines the behaviour of people in labour.

Despite the large number of studies investigating the sources and mechanism of expression of satisfaction, there is no consensus on the issue of its content and measurement. Most of the studies are based on a variety of existing theories of motivation. Quantification of satisfaction is a difficult process, so nonparametric methods for establishing its level can be used in practice. The processing of the received data can be performed using a variety of computer programs for data analysis that allow the establishment of links and dependencies, verification of hypotheses and prediction of future trends. This paper presents the results of the research on motivation for learning and specific military activities in the learning process which ensued from the first question - "Which is the measure for your satisfaction with the choice of academic specialty?/How satisfied are you with your choice of academic specialty?". The cadets who graduated in the academic years 2013/2014, 2014/2015, and 2015/2016, were the basic target group that was interviewed. First-year cadets and students who started their training in the academic year 2013/2014 were included as control groups for comparison.

Cadets' answers to the question are as indicated below. 


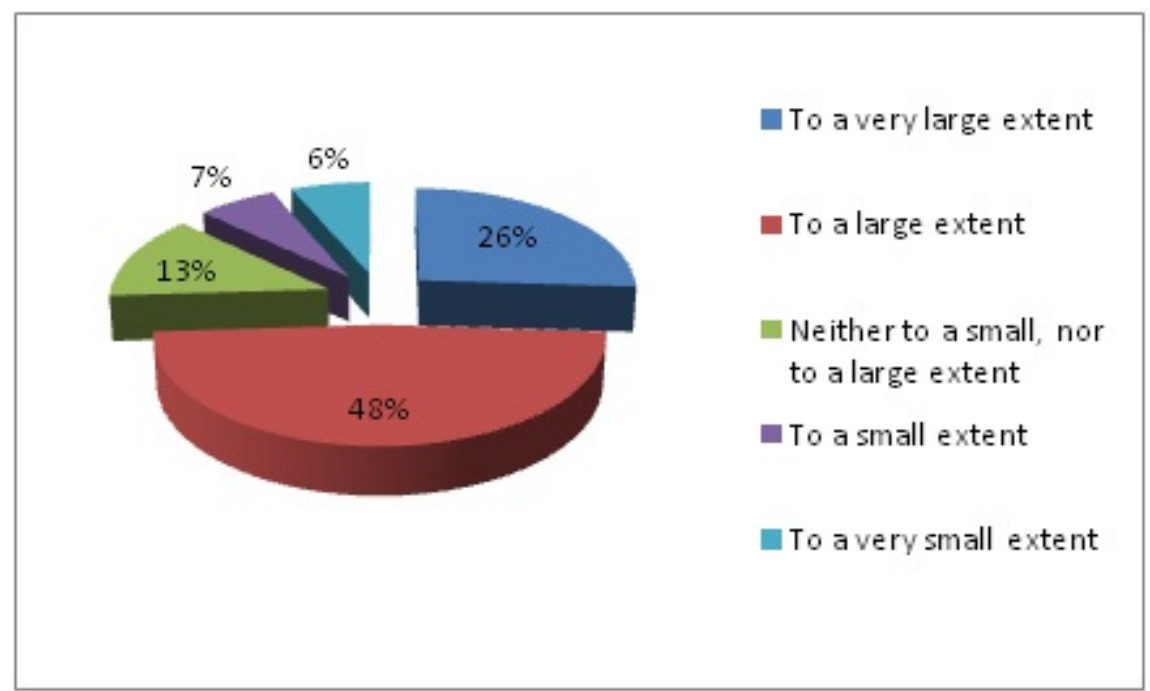

Figure 1: Which is the Measure for Your Satisfaction with the Choice of Academic Specialty? Cadets 1st Academic Year of Training2013/2014 Academic Year

Figure 1 shows the following distribution $26 \%$ of the respondents, who are cadets in their first year of training, are satisfied with the specialty to a very large extent, and $48 \%$ of the respondents are satisfied with the specialty to a large extent, which shows a significant percentage of satisfaction $74 \%$ of the respondents are satisfied with their choice of academic specialty. Indifference is expressed by $13 \%$ of the respondents, which is quite a significant result. $7 \%$ of the respondents are satisfied with their choice of academic specialty to a small extent and $6 \%$ of the respondents are satisfied to a very small extent.

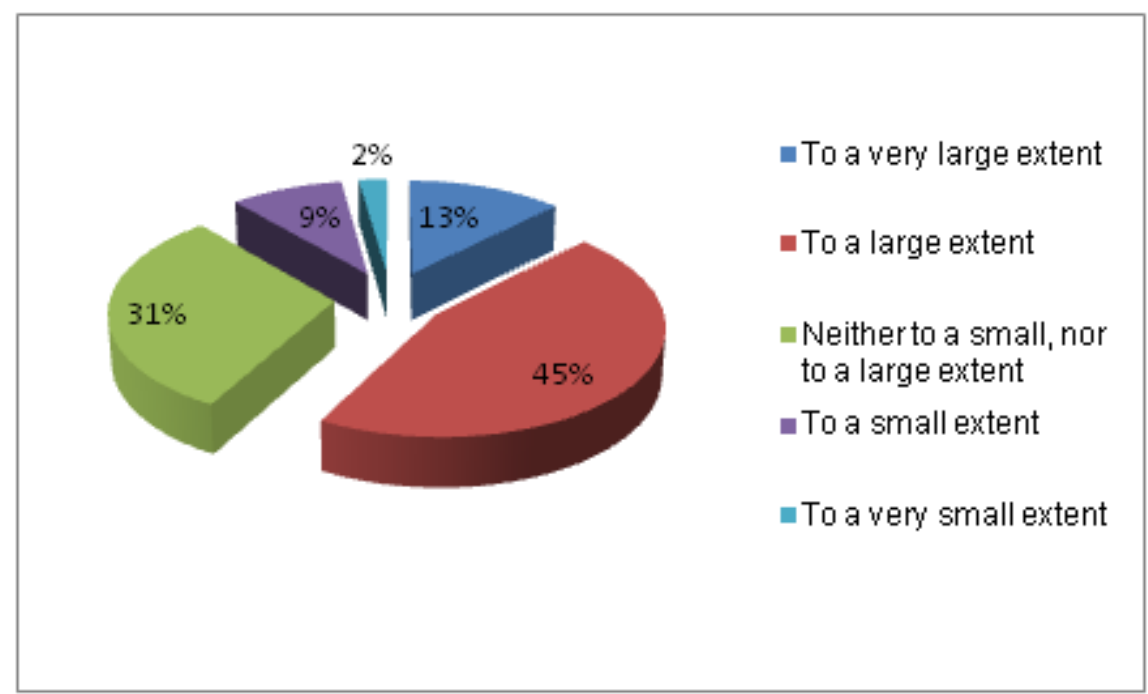

Figure 2: Which is the Measure for Your Satisfaction with the Choice of Academic Specialty?Students 1st Academic Year of Training2013/2014 Academic Year

Figure 2 shows the following distribution $13 \%$ of the surveyed first-year students are satisfied with their choice of specialty to a very large extent, and $45 \%$ of the respondents are satisfied with the specialty to a large extent, which shows a total $58 \%$ of satisfaction with the chosen specialty. Indifference is expressed by $31 \%$ of the respondents, which is quite a significant result. The satisfied with the chosen 
specialty to a small extent are $9 \%$ of the respondents, and to a very small extent are satisfied $2 \%$ of the respondents.

The data analysis shows that the satisfaction of cadets in their first academic year of training is significant, while students' results show a remarkably lower satisfaction. We can find notable differences between the two control groups of cadets and civilian students. They are presented in the figure below.

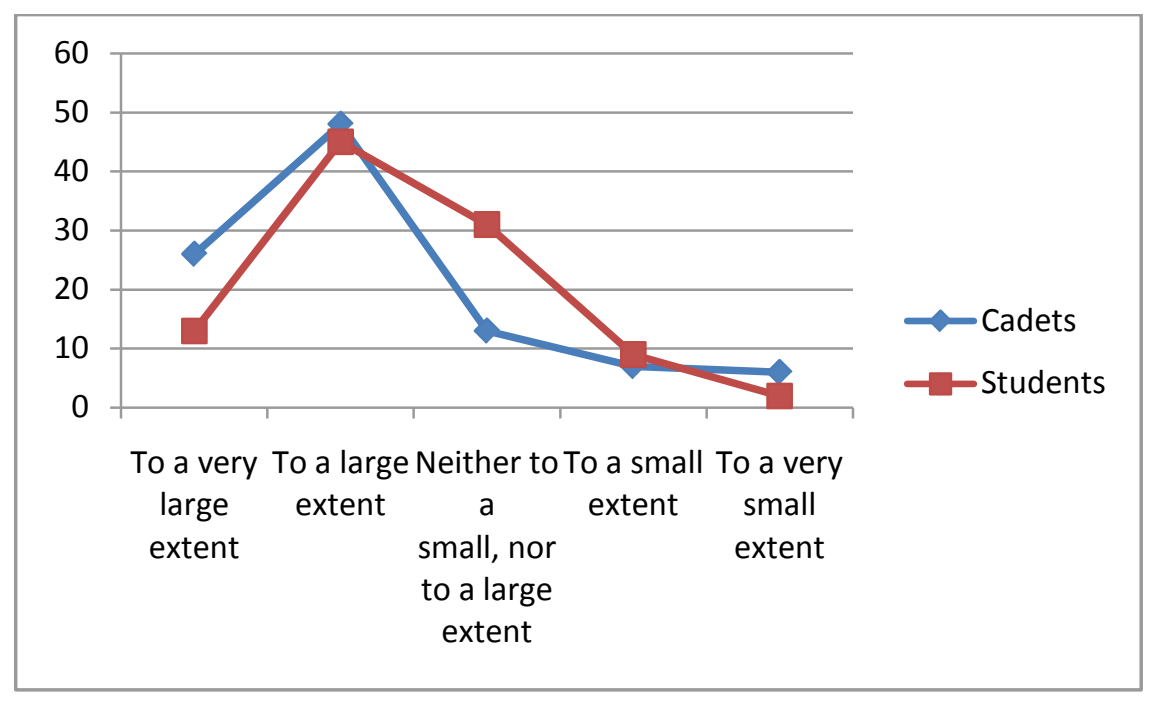

Figure 3:Comparison of Measuring Satisfaction with the Choice of Academic Specialty - Control Groups

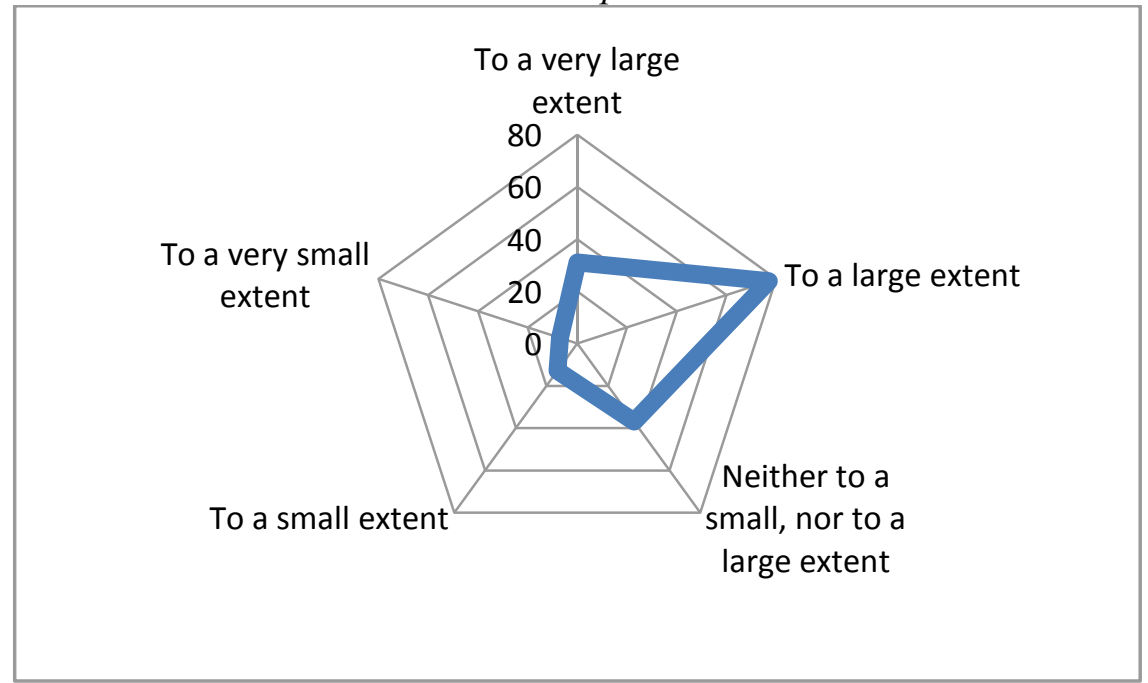

Figure 4:Measuring Satisfaction with the Choice of Academic Specialty - Control Groups - General Presentation

The data analysis shows that more than half of the respondents are satisfied with their choice of academic specialty, and frustration is at low rates. The comparative analysis reveals significant differences in the responses of the control groups of students and cadets and the studied classes of cadets. They are presented in the table below. 
Table 1 Levels of Satisfaction with the Choice of Academic Specialty/all studied groups of

respondents/

\begin{tabular}{|l|c|c|c|}
\hline \multicolumn{1}{|c|}{ Respondents } & Satisfaction, $\%$ & Indifference, $\%$ & Dissatisfaction, $\%$ \\
\hline $\begin{array}{l}\text { Cadets 1st academic year } \\
\text { 2013/2014 }\end{array}$ & 74 & 13 & 13 \\
\hline $\begin{array}{l}\text { Students 1st academic year } \\
\text { 2013/2014 }\end{array}$ & 58 & 31 & 11 \\
\hline Alumni Cadets 2013/14 & 85 & 13 & 2 \\
\hline Alumni Cadets 2014/15 & 73 & 21 & 6 \\
\hline Alumni Cadets 2015/16 & 68 & 24 & 8 \\
\hline
\end{tabular}

The highest level of satisfaction with the specialty is expressed by the cadets who graduated in 2013/2014. The data which from the control group of cadets and cadets who graduated in 2014/2015 are close in value.[3] The control group of students in their first academic year of training show the lowest satisfaction with the specialty. At the same time, the same respondents, namely the control group of students in their first academic year of training, show great indifference and frustration. These data show the importance of military organisational culture and the difficulties that students have in adapting in the initial stage of their education.
The test indicator, namely satisfaction with the choice of academic specialty, confirms the research hypothesis that the specific organisational culture at the military university has a major impact on individual motivation for learning. The data direct the study to the need for analysing in detail the results of an empirical research on learners' motivation, establishing the major problem areas in the main target group of respondents and in the control groups for comparison, and formulation of recommendations for developing a program for improving motivation for education by offering activities and events to raise it.

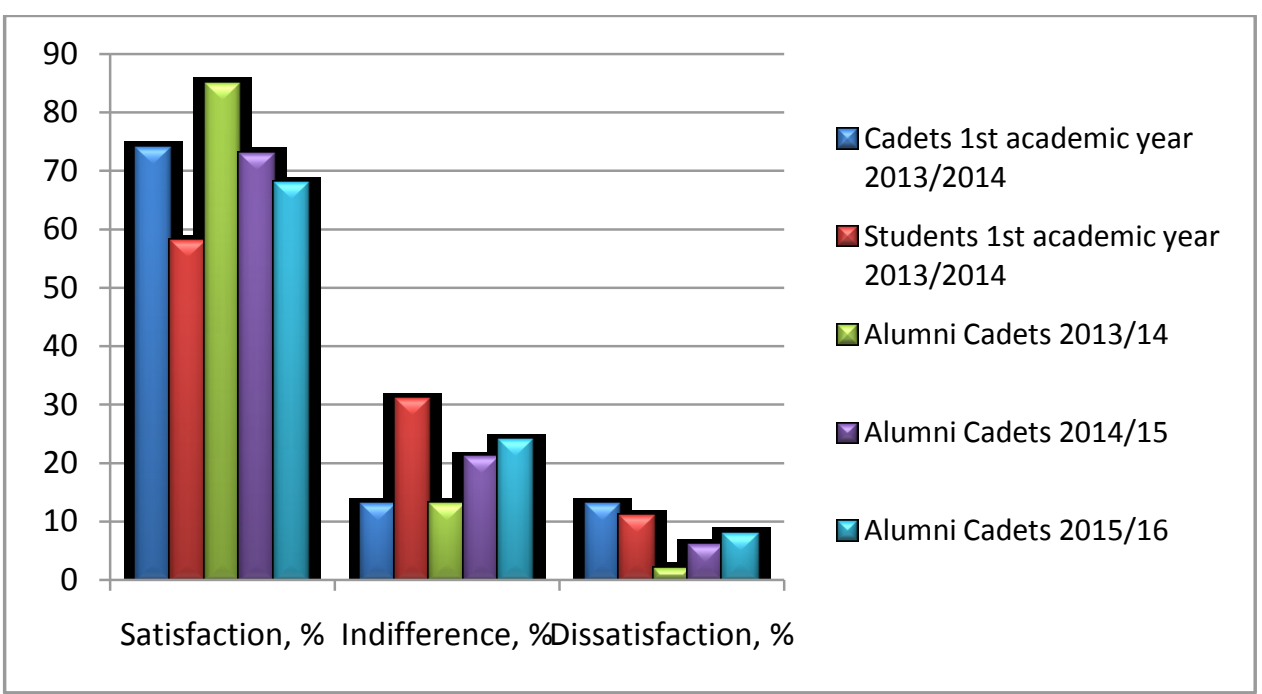

Figure 5: Comparison of the Levels of Satisfaction with the Choice of Academic Specialty

\section{Conclusion}

In order to clarify in detail the cadets' and students' motivation for education and their satisfaction, the following areas could also be explored: studying specific subjects and topics and their relationship with the professional development of cadets and civilian students as future leaders; rigor of 
the academic program and its relationship to the academic achievements of cadets and civilian students; personal participation of cadets and civilian students during seminars and exercises; connection between learning process and future realisation of cadets and civilian students as good military commanders or good civilian managers; different methods for motivation used by academic or command staff and their influence on the behaviour and motivation of cadets and civilian students in the learning process; impact of military organisational culture on individual performance.

\section{References}

[1] Manolov, D.,Directions for Improving the Planning of the Supply with Clothes, Shoes and Equipment in the Bulgarian Army, ISBN 978-973-153-245-5, the $22^{\text {nd }}$ International Scientific Conference, KBO, Sibiu, Romania, 2016.

[2] Nichev, N., Petrova, E., Professional Training of Future Logistic Officers at the National Military University of Bulgaria, Sibiu, Romania, The 21st International Conference The Knowledge-Based Organization, NicolaeBalcescu Land Forces Academy, 11-13 June 2015, pp. 259 $\div$ 263, ISSN 1843-6722.

[3] Petrova, E., A Study on the Cadets' Satisfaction with Choice of Academic Specialty at the National Military University, Bulgaria, Socio-economic Analysis Scientific Journal,Department of Economics, St. St. Cyril and Methodius University, Bulgaria, 2016 / Volume 8 / Number 1, ISSN: 2367-9379, pp. $38 \div 43$.

[4] Petrova, E., Basics of Management, Publishing complex of National Military University, Bulgaria, VelikoTarnovo, 2014, ISBN 978-954-753-121-5.

[5] Petrova, E., Brief Introduction to Management, Publishing complex of National Military University, Bulgaria, VelikoTarnovo, 2015, ISBN 978-954-753-120-8.

[6] Petrova, E., Management in a Changing World, Publishing complex of National Military University, Bulgaria, VelikoTarnovo, 2012, ISBN 978-954-753-093-5.

[7] Petrova, E., Methodology on Study of Motivation for Education based on Opinions' Research of Cadets fromVasilLevski National Military University, Bulgaria, Socioeconomic Analysis Scientific Journal,Department of Economics, St. St. Cyril and Methodius University, Bulgaria, 2015Book 2/ Volume8/ISSN: 2367-9379, pp. $127 \div$ 133.

[8] Petrova, E., Specific Subjects of License Academic Program - an Important Stage of Professional Development of Future Military Leaders at the National Military University, Bulgaria, The Annals of SpiruHaret University, Romania, Volume 6, issue 3, 2016, ISSN 2393-1795, ISSN-L 2068-6900, pp.113 $\div 119$.

[9] Petrova, E., Zegoicea, Andrei, Comparative Case Study Regarding Cadet's Motivation - Methodology, RevistaAcademieiFortelorTerestre,NicolaeBalcescu Land Forces Academy,Sibiu, Romania, Vol. XVIII, Nr. 3 (71), Trimestrul III, 2013, pp. $301 \div 307$. 